\title{
Temperature Dependence of a Dielectric Relaxation in Weakly Polar Ferrofluids
}

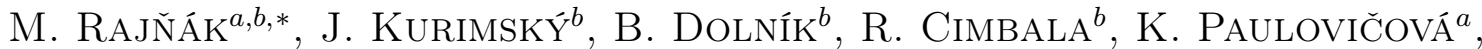 \\ P. KOPČANSKÝ ${ }^{a}$ AND M. TIMKO ${ }^{a}$ \\ ${ }^{a}$ Institute of Experimental Physics, SAS, Watsonova 47, 04001 Košice, Slovakia \\ ${ }^{b}$ Faculty of Electrical Engineering and Informatics, Technical University of Košice, Letná 9, 04200 Košice, Slovakia
}

\begin{abstract}
In this paper, we report on the temperature dependent broadband dielectric response of a ferrofluid based on transformer oil and magnetite nanoparticles covered with oleic acid molecules. For that purpose the method of dielectric spectroscopy has been chosen in the frequency range from $20 \mathrm{~Hz}$ up to $100 \mathrm{kHz}$. The experiments were carried out on thin film ferrofluid samples confined in a glass plate capacitor containing indium tin oxide (ITO) plate electrodes. The obtained complex permittivity spectrum shows a pronounced dielectric dispersion in the low frequency range. Taking into account the ferrofluid composition we associate this relaxation with ion impurity polarization at the nanoparticle-oil interface. The strong temperature dependence of the relaxation process has been found when conducting the experiments in the temperature range from $298 \mathrm{~K}$ to $358 \mathrm{~K}$. The relaxation time of the revealed process exhibits a typical Arrhenius behavior. Based on the conducted experiments and analysis, we propose some reasonable practical applications of the studied ferrofluid in the field of electrical engineering.
\end{abstract}

DOI: 10.12693/APhysPolA.131.943

PACS/topics: 47.65.Cb, 77.22.Ch, 77.22.Gm, 82.70.Dd

\section{Introduction}

Transformer oils (TO) are a major part of the electrical insulation system in many types of electrical equipment. Besides the electrical insulation, they provide effective heat transfer, acting as a coolant. To increase the lifetime and reliability of electrical equipment it is necessary to decrease its operating temperature. For that purpose, researchers look for additives enhancing the TO's thermal properties and retaining their excellent insulating characteristics. The superparamagnetic nanoparticles (SNP) [1] are promising additives, whose stable dispersions in TO constitute ferrofluids with a potential application in electrical engineering. The intuitively enhanced cooling ability of the resulting ferrofluid originates primarily in the increased thermal conductivity due to the particles $[2,3]$. Moreover, in such a liquid coolant the effect of thermomagnetic convection has been observed when exposed to temperature and magnetic field gradients, which are inherently present around power transformers $[4,5]$. These thermal properties of transformer oil based ferrofluids can thus enhance loadability of power and related transformers. From the dielectric point of view, it is well known that the presence of SNP in TO increases their dielectric permittivity [6], and can even increase their dielectric breakdown field strength [7]. As the former effect is quite intuitive, the latter is paradoxical and requires comprehensive experimental investigation of the ferrofluids in both, high and low electric fields. Hence, the investigation of novel transformer oil-based ferrofluids has to

*corresponding author; e-mail: rajnak@saske.sk involve the analysis of the ferrofluid dielectric response, especially in a broad frequency range. The frequency response of dielectric liquids is defined essentially by the manner in which complex dielectric permittivity varies with frequency, temperature, and applied electric field as parameters [8]. Consequently, the permittivity behavior permits to establish the character of the loss mechanism under a given set of conditions. Also, the permittivity spectrum allows some important deductions to be made as regards the chemical and physical structure of the ferrofluid which can be essential for the streamer development. Thus, even though power transformers operate at 50 or $60 \mathrm{~Hz}$ frequency, it is desirable to investigate the ferrofluids' dielectric properties in a broader frequency range, too. Herein we present the temperature dependent dielectric response of a thin layer of a transformer oil-based ferrofluid within the frequency range from $20 \mathrm{~Hz}$ up to $100 \mathrm{kHz}$, measured by an LCR meter.

\section{Materials and methods}

The investigated ferrofluid was prepared in a well proven way [9]. Firstly, magnetite $\left(\mathrm{Fe}_{3} \mathrm{O}_{4}\right)$ nanoparticles were co-precipitated from aqueous solution of $\mathrm{Fe}^{2+}$ and $\mathrm{Fe}^{3+}$ ions in the presence of $\mathrm{NH}_{4} \mathrm{OH}$ at $80-82{ }^{\circ} \mathrm{C}$. Then, the nanoparticles were sterically stabilized with a single vegetal oleic acid layer $\left(\mathrm{C}_{18} \mathrm{H}_{34} \mathrm{O}_{2}, 65-88 \%\right.$, Merck), chemisorbed on the particle surfaces. The chemisorption was followed by washing with distilled water with magnetic decantation to remove residual unreacted salts. Then, the flocculation re-dispersion procedure was repeated several times to ensure a negligible presence of free surfactant in the final solution. The purified magnetite nanoparticles were dispersed in the inhibited mineral oil 
(MOGUL TRAFO CZ-A, PARAMO) with a particle volume fraction equaled to $6.6 \%$. This value was determined from magnetization measurements as a ratio of the ferrofluid magnetization of saturation $\left(29.6 \mathrm{kA} \mathrm{m}^{-1}\right)$ to the domain magnetization of bulk magnetite $\left(446 \mathrm{kA} \mathrm{m}^{-1}\right)$. To study the ferrofluid dielectric response we used the LCR meter Agilent E4980A with the frequency range from $20 \mathrm{~Hz}$ up to $2 \mathrm{MHz}$. The sample was confined into a gap of a small capacitor with ITO electrodes separated $1.9 \mu \mathrm{m}$ apart. Measurements were performed with the applied test voltage of $38 \mathrm{mV}$. To vary the sample temperature the capacitor was inserted inside a temperature controller (Linkam Scientific Instruments). The measurements were performed at temperatures from $298 \mathrm{~K}$ to $358 \mathrm{~K}$, stabilized within $0.1 \mathrm{~K}$.

\section{Results and discussion}

By the above mentioned method we measured capacitance $(C)$ and dissipation factor $(\tan \delta)$ of the sample in the parallel-plate capacitor, which are defined as follows:

$$
\begin{aligned}
& C(\omega)=\varepsilon_{0} \varepsilon^{\prime}(\omega) S / d, \\
& \tan \delta(\omega)=\varepsilon^{\prime \prime}(\omega) / \varepsilon^{\prime}(\omega),
\end{aligned}
$$

where $S$ and $d$ are the sample cross-sectional area and thickness, respectively, and $\varepsilon_{0}$ is the permittivity of vacuum. Such measurements make available the frequency dependence of the parameters $\varepsilon^{\prime}$ and $\varepsilon^{\prime \prime}$, which are, respectively, the real and imaginary parts of the (relative) complex dielectric permittivity function [8]:

$$
\varepsilon^{*}(\omega)=\varepsilon^{\prime}(\omega)-\mathrm{i} \varepsilon^{\prime \prime}(\omega),
$$

where $\mathrm{i}^{2}=-1$. The spectra of both parameters as functions of the sample temperature are presented in Fig. 1. As the finite ITO electrode resistance causes a spurious signal emerging in the dielectric spectrum as dispersion above $100 \mathrm{kHz}$, we present the data below this boundary, so focusing on the real sample behavior. From the presented spectra one can see a pronounced low frequency relaxation process. To analyze this spectrum we take into account that the transformer oil shows constant permittivity $(\varepsilon=2.1)$ in the whole frequency range. As the known conductivity and permittivity values of the sample constituents determine the appearance of the MaxwellWagner relaxation at much higher frequencies, we associate the low frequency process with the ion impurity polarization taking place on the SNP surfaces [10, 11], following the famous Schwarz polarization model.

Concerning the shape of the loss spectra one can conclude that the relaxation maximum resemble to a Debyelike dielectric relaxation mode. The simple Debye-like dielectric behavior can be also deduced from the Cole-Cole presentation depicted from the permittivity values taken at the highest temperature, which is shown in Fig. 2. The experimental data tend to create a semicircle with its centre on the horizontal axis. In general, the semicircle character is considered as the main characteristic of a Debye relaxation process. To verify the Debye-like relaxation behavior we applied the Debye fitting function:
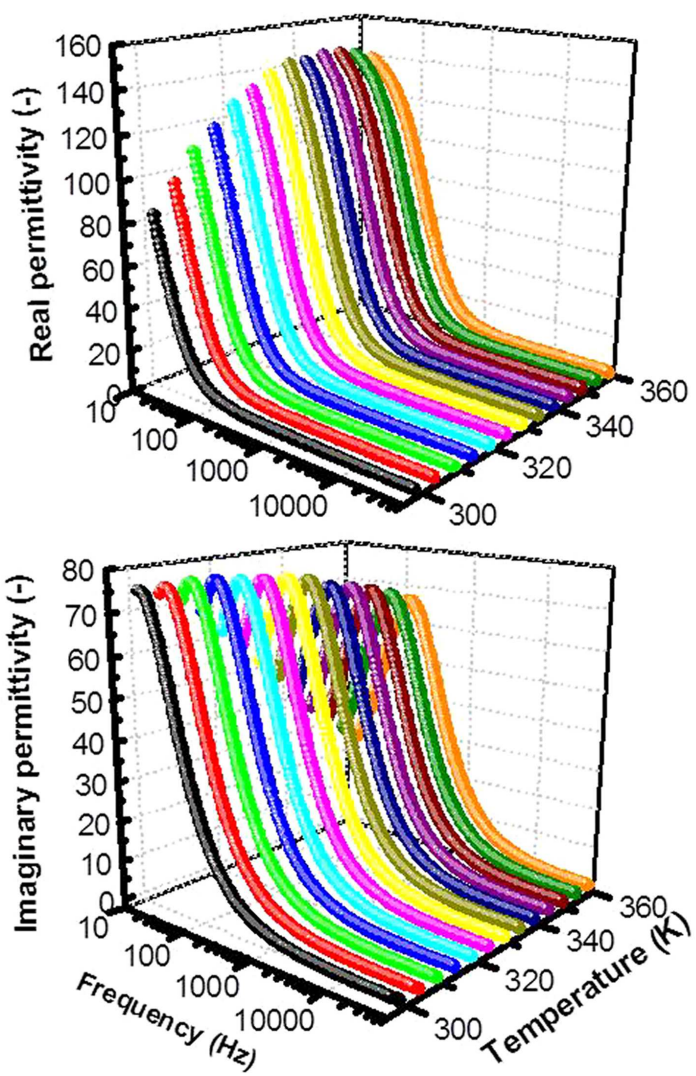

Fig. 1. Temperature dependence of the real (upper graph) and imaginary permittivity (bottom graph) spectra.

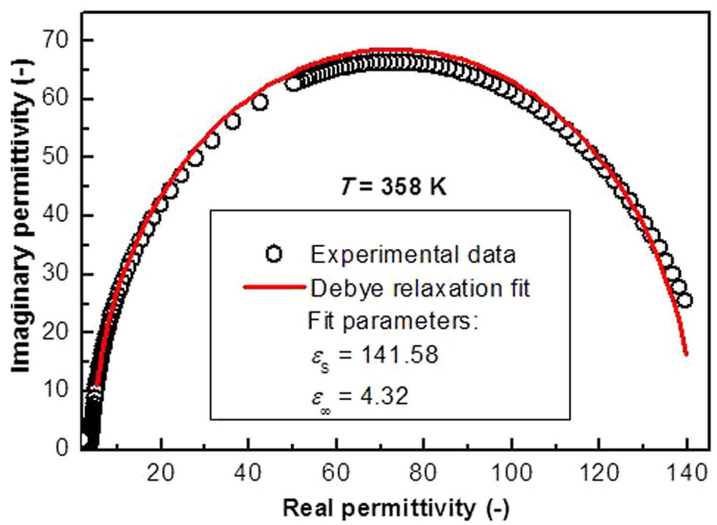

Fig. 2. The complex plane plot (Cole-Cole) of the measured ferrofluid permittivity.

$$
\left(\varepsilon^{\prime}-\frac{\varepsilon_{S}-\varepsilon_{\infty}}{2}\right)^{2}+\varepsilon^{\prime \prime 2}=\left(\frac{\varepsilon_{S}-\varepsilon_{\infty}}{2}\right)^{2}
$$

The resulting fitting parameters represent the static permittivity $\varepsilon_{S}$, and the high frequency limit of the real permittivity $\varepsilon_{\infty}$ with the found values of 141.58 and 4.32 , respectively. However, a small deviation in the experimental data from the theoretical Debye fitting function is observed especially in the low frequency region. This deviation can be associated with the particle size distri- 
bution and the related distribution of relaxation times. Moreover, the dielectric losses due to the space charge relaxation (migration) in the sample and a small contribution from the DC conductivity may slightly influence the low frequency dielectric response.

From Fig. 1 it can be noticed that the relaxation maximum moves towards higher frequencies with increasing temperature. Thus, the relaxation time of the related process decreases with increasing temperature. We obtained the particular relaxation times of the considered process from the Havriliak-Negami fitting function

$$
\varepsilon^{*}(\omega)=\varepsilon_{\infty}+\frac{\varepsilon_{S}-\varepsilon_{\infty}}{\left[1+(\mathrm{i} \omega \tau)^{\alpha}\right]^{\beta}},
$$

where $\alpha$ and $\beta$ are empirical exponents and $\tau$ is the relaxation time. The fitting of the dielectric spectra at constant temperature yielded the relaxation time values, which are depicted in the Arrhenius plot in Fig. 3. From this figure it is clearly seen that the logarithm of the relaxation time linearly decreases with increase of temperature. Therefore, the relaxation time decreases exponentially with temperature in accordance to the famous Arrhenius law

$$
\ln \tau(T)=\ln \tau_{0}+\frac{E_{A}}{R T},
$$

where $\tau_{0}$ is a pre-exponential factor, $R$ is the universal gas constant, $T$ is the absolute temperature and $E_{A}$ is the activation energy. From the fit it follows that the value of the activation energy $E_{A}$ is $27.833 \mathrm{~kJ} \mathrm{~mol}^{-1}$.

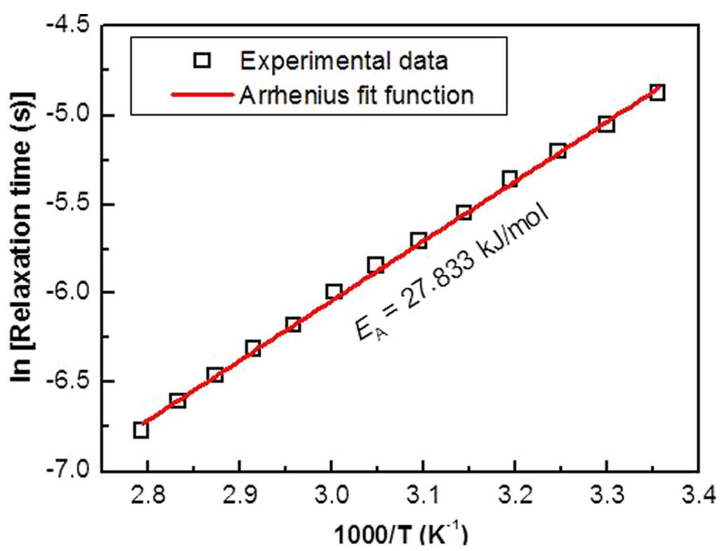

Fig. 3. Arrhenius plot of the temperature dependent low frequency relaxation process in the studied ferrofluid.

\section{Conclusions}

Spectroscopic measurements of the complex dielectric permittivity of the ferrofluid based on the transformer oil Mogul revealed the Debye-like relaxation process. This relaxation has been associated with the ionic impurities polarization on the particle surfaces. Nearly Debye-like behavior has been confirmed by fitting the complex plane permittivity data. The analysis of the temperature dependence of the detected low frequency relaxation follows the Arrhenius law. From the practical application point of view, the highest dielectric losses observed around commonly operating frequencies $(50 \mathrm{~Hz})$ could signify a possible drawback when applying this ferrofluid in high voltage power transformers. On the other hand, this ferrofluid can find potentially reliable use as an insulating medium in pulsed power systems or in high voltage direct current transmission systems.

\section{Acknowledgments}

This work was supported by Slovak Academy of Sciences and Ministry of Education: VEGA 2/0016/17, 1/0311/15, 2/0141/16, 1/0377/16, Ministry of Education Agency for structural funds of EU, Project No. 26220120046, 26220120055, 26220120033 and Slovak Research and Development Agency under the contract No. APVV-15-0438. We acknowledge Dr. Elena Alina Taculescu-Moaca for preparing the investigated ferrofluid.

\section{References}

[1] An-Hui Lu, E.L. Salabas, F. Schuth, Angew. Chem. 46, 8 (2007).

[2] I. Nkurikiyimfura, Y. Wang, Z. Pan, Renew. Sustain. Energy Rev. 21, 548 (2013).

[3] M. Lajvardi, J. Moghimi-Rad, I. Hadi, A. Gavili, T. Dallali Isfahani, F. Zabihi, J. Sabbaghzadeh, J. Magn. Magn. Mater. 322, 21 (2010).

[4] S.A. Suslov, Phys. Fluids 20, 8 (2008).

[5] S. Banerjee, A. Mukhopadhyay, S. Sen, R. Ganguly, Numer. Heat Transf. Part Appl. 59, 693 (2011).

[6] M. Rajnak, M. Timko, L. Tomco, K. Marton, J. Kurimsky, B. Dolnik, R. Cimbala, M. Molcan, P. Kopcansky, Magnetohydrodynamics 49, 3 (2013).

[7] J.Ch. Lee, H.S. Seo, Y.J. Kim, Int. J. Therm. Sci. 62, 29 (2012).

[8] F. Kremer, A. Schönhals, Broadband Dielectric Spectroscopy, Springer, Berlin 2003.

[9] L. Vékás, D. Bica, M.V. Avdeev, China Particuology 5, 43 (2007).

[10] M. Rajnak, J. Kurimsky, B. Dolnik, K. Marton, L. Tomco, A. Taculescu, L. Vekas, J. Kovac, I. Vavra, J. Tothova, P. Kopcansky, M. Timko, J. Appl. Phys. 114, 034313 (2013).

[11] M. Rajnak, J. Kurimsky, B. Dolnik, P. Kopcansky, N. Tomasovicova, E.A. Taculescu-Moaca, M. Timko, Phys. Rev. E 90, 032310 (2014). 